\title{
La crónica deportiva: José A. Sánchez Araujo
}

\author{
Joaquín M. Marín Montín \\ Licenciado en Ciencias de la Información. Investigador de Comunicación y \\ Deporte. Universidad de Sevilla
}

\section{RESUMEN}

Los medios de comunicación y el deporte moderno están íntimamente ligados desde su nacimiento en el pasado siglo. Primero fueron los periódicos los testigos de la actividad deportiva, posteriormente la radio, alcanzando el desarrollo pleno con la televisión. Ninguna otra actividad humana atrae tanta expectación y esto hace del periodista deportivo una figura muy importante en el mundo actual, siendo el puente entre el público y el deporte. La crónica constituye uno de los géneros más utilizados en la prensa escrita deportiva. El fútbol y José Antonio Sánchez Araujo, sería uno de los mejores ejemplos y objeto de este análisis.

\section{ABSTRACT}

Media and Sport are intimately bound from their birth in last century. In the beginning, newspapers were witness of sport activity, reaching the full development with radio and television. No other human activity attracts so much expectation and this makes of sport journalist a very important figure in current world, being the bridge between public and sport. The chronicle constitutes one of the goods more used in sport press. This article analyses soccer in media and the figure of a sport journalist in Spain called José Antonio Sánchez Araujo.

Palabras claves: Medios de comunicación/Deporte/Crónica Deportiva/Periodista deportivo/José Antonio Sánchez Araujo

Key Words: Media/Sport/Sport chronicle/Sport journalist/José Antonio Sánchez Araujo.

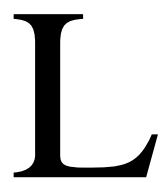

os géneros del periodismo se emplean en la sección de deportes de los distintos medios en la misma medida que en las restantes facetas comunicativas. Así podemos hablar además de las crónicas, de comentarios, críticas, artículos y entrevistas. Uno de los rasgos más característicos de esta sección en cuanto a su estilo, independientemente de la calidad literaria de cada medio y periodista, será el lenguaje particular del deporte. La expresiones son generalmente de origen belicista, como corresponde a "una actividad propulsora

1 ALCOBA, Antonio: Deporte y comunicación, Dirección General de Deportes de la Comunidad Autónoma de Madrid, Madrid, 1984, p.147 
de enfrentamientos entre una o más personas", como señala Antonio Alcoba. ${ }^{1}$ Es habitual el uso de modismos y muletillas extraídas de la parcela militar, mezclándose con palabras técnicas del deporte, imprescindibles a la hora de relatar un partido, una carrera o en general cualquier evento deportivo.

Esa idea no significa que el género periodístico, en su aspecto más puro no se emplee: pero el destinatario de la actividad deportiva lo que busca en una crónica, un comentario, una entrevista o un artículo, es la aproximación más fidedigna posible a lo sucedido o a lo que puede acontecer. Para ello el periodista deportivo precisa del conocimiento de la terminología o lenguaje propio de cada disciplina deportiva y de argumentos a través de los cuales puede hacer llegar la emoción de la lucha deportiva, el ambiente del juego,...

A la hora de escribir, hablar o realizar audiovisualmente deporte, lo que realmente quiere el lector, oyente o espectador es una explicación de lo acontecido en el estadio, cancha, piscina, terreno de juego,... lo más simple pero a la vez lo más completa posible. Es probable que desde el punto de vista literario el deportivo no es precisamente un género donde se empleen al máximo los recursos, ya que en el caso de la prensa escrita en cuarenta o cincuenta líneas debe ofrecerse el relato de una competición de hora y media de duración si hablamos de fútbol y no pueden hacerse muchas concesiones a la galería, teniendo que ir al grano.

Al ser un tipo de información dirigida a toda clase de público masivo como lo demuestran los datos, para que todos se enteren, es necesaria la sencillez en el relato. Aunque introducir en él la terminología apropiada, junto con apuntes técnicos y anecdóticos, eso supone el mejor atractivo para el lector del mensaje deportivo. En este sentido el ejemplo que nos ocupa de José A. Sánchez Araujo cumple perfectamente dicho requisito.

\section{Delimitación del género en el deporte}

La definición del género de la crónica deportiva podríamos considerarla como la narración de un hecho de forma continuada, si nos atendemos a su origen etimológico. La palabra crónica tiene un origen histórico-literario antes de su adaptación al periodismo tal y como hoy la conocemos. Su origen se remonta a los relatos de la época clásica, en la que griegos y romanos contaban hechos bélicos, narrados por sus propios protagonistas o testigos de los mismos, con un orden temporal. ${ }^{2}$

Por su propia etimología, estamos ante relatos en el que se observa una sucesión temporal de acontecimientos, con un hilo conductor. Relatos cronológicos referidos lo sucedido entre un periodo delimitado (hora, día, semana,...). Bernal Rodríguez la entiende como "una información de hechos noticiosos, ocu-

2 MARTÍN VIVALDI, G: Géneros periodísticos. Paraninfo, S.A. Madrid, 1981, p.123 
rridos en un periodo de tiempo, por un cronista que los ha vivido como testigo, investigador, e incluso, como protagonista y que al mismo tiempo, que los narra, los analiza, e interpreta, mediante una explicación personal". Y continúa " El cronista suele ser un experto que realiza su labor con continuidad desde el propio escenario de los hechos o sus inmediaciones". ${ }^{3}$ En deporte, será por tanto, el seguimiento y relato de una competición deportiva, de un equipo o deportista.

Frente a otros géneros periodísticos, la crónica en el deporte permite cierta libertad a la hora de escribir o hablar de eventos deportivos, al no precisar ceñirse de manera estricta a la actividad deportiva.

La crónica de un acontecimiento de esta índole supone un respiro en el sentido de poder abordar otros temas sin salirse del principal motivo. Es por tanto normal, hacer una semblanza de la ciudad, zona o país donde tiene lugar el suceso deportivo y comentarse aspectos que pueden ser desconocidos, con el objetivo de introducir en el escenario de la competición a lectores o receptores del medio en general. Todo ello faculta al periodista deportivo, a tomarse la libertad de exponer en estilo diferente al normal, cuestiones fuera de la temática deportiva. En cualquier caso el periodista deportivo deberá ser un buen conocedor y sobre todo entusiasta del deporte y como señala Alcoba "y si es o ha sido practicante mejor que mejor, pues de su experiencia, puede extraer la base para redondear su formación periodística". ${ }^{4}$

Las crónicas deberán ser atractivas y presentar a los receptores de los medios aspectos diferentes a los habituales en el tratamiento del deporte que trate en cuestión. Aquí podrían entrar datos y estadísticas del evento, recordándose así mismo hechos y curiosidades. Ello no impide, dentro del texto, informar del seguimiento realizado a equipos y deportistas en sus entrenamientos y los momentos de ocio, sin olvidar añadir las anécdotas que hayan podido surgir alrededor de todo el ambiente deportivo.

La crónica es un género de escape para dar rienda suelta, sin exagerar, a las virtudes literarias y culturales del periodista deportivo, algo que agradecen los receptores de estos mensajes, al proporcionarles una visión diferente y complementaria a la actividad deportiva. En el caso de las crónicas de fútbol editadas en prensa, ¿podrían serlo tras la visión en televisión o radiodifusión, como análisis reflexivo del día siguiente?

Como cualquier periodista, el especialista en deporte, debe ser consecuente con el código ético y deontológico, a través del cual se reconozca su imparcialidad. A juicio de Alcoba el periodista deportivo es, ante todo, un observador

3 BERNAL RODRIGUEZ, M.: La crónica periodística, tres aproximaciones a su estudio. Padilla Libros Editores y Libreros, Sevilla. 1997, p.27

4 ALCOBA, Antonio: Cómo hacer periodismo deportivo, Editorial Paraninfo, Madrid, 1993, p.45. 
imparcial con la obligación de estar preparado para ofrecer una opinión sincera y realista. Pese a todo la crónica, tendrá una carga subjetiva importante, que dependerá de cada caso y circunstancia que haya acontecido en cada partido.

\section{Evolución de las crónicas deportivas}

$\mathrm{Su}$ estructuración y forma de representación han evolucionado con el paso del tiempo. La prensa escrita fue la que inició la parcela deportiva en sus páginas. La aparición de este género específico se debió a la importancia cada vez mayor adquirida por el deporte a finales de Siglo XIX, y sobre todo en la primera mitad del XX hasta alcanzar el auge actual. Según Alcoba puede que el interés por la información deportiva tuviera uno de sus elementos más importantes en la restauración de los Juegos Olímpicos. En los primeros de ellos de la era moderna celebrados en Atenas en 1896, entre los setenta mil espectadores, testigos de las competiciones, se encontraban los corresponsales del diario parisino Le Figaró y el londinense The Times, empezándose a publicar ya las primeras crónicas sobre deportistas y resultados. ${ }^{5}$

El deporte fue introducido en los diarios al darse cuenta los propietarios de los periódicos del interés que dicha actividad despertaba en los ciudadanos. En la primera mitad del Siglo XIX aparece en Londres el primer diario especializado en deportes Sportman, denominado más tarde Sporting Life. En 1892 en Francia surge el primer diario deportivo, Le Veló. En España hasta 1906 no aparece el primer diario especializado, El Mundo deportivo. Sobre esta época surgirán también otras publicaciones como La Semana Madrileña, El Sport Español, Crónica del Sport o La Revista Ilustrada de Sports. Los primeros informadores de temas deportivos en los periódicos no fueron periodistas, sino escritores aficionados a un fenómeno incipiente, que realizaban comentarios con el estilo propio de la época, retórico y muy floreado. A finales del pasado siglo la crónica deportiva formaba parte de los sueltos incluidos en los ecos de sociedad, con una extensión no superior a las veinte líneas y con un contenido que se limitaba a reseñar el campo, el árbitro, las alineaciones y autores de los goles en el caso del fútbol. Léxicamente dominaba la presencia de anglicismos, relacionados con la jerga de la disciplina deportiva.

En la década de los diez se añade a la crónica una valoración de la actuación individual de los jugadores. Siguen dominando los anglicismos puros. En la los años siguientes, los veinte, se adoptan modelos de crónicas extranjeros con un estilo divulgador, en el que se produce una primera reducción de anglicismos. No se usan recursos tipográficos que separen las distintas partes de las crónicas, a 
excepción de la distinción del titular y el cuerpo de la crónica. A partir de 1926 se adopta el modelo francés para mezclar la objetividad y la subjetividad. El modelo francés es una forma mixta entre los contenidos realistas del deporte y el enlace con sus aspectos superficiales para dar amenidad. Entre 1938 y 1956 se creará un patrón de crónica español, que adopta formas del modelo francés y que se caracteriza por eliminar extranjerismos y por enlazar los aspectos superficiales de la competición deportiva con las formas culturales despolitizadas impuestas por el gobierno franquista y con valores históricos.

En la actualidad, el cronista no sigue una estructura interna fija, la crónica actual es un género con unas características morfológicas y sintácticas propias. Presentan como principales rasgos léxicos la formación de subcódigos que recogen cambios de sentido de términos, procedentes de otras áreas sociales, discurso repetido y aspectos de retórica. En España se ha generalizado una fórmula que utilizan las publicaciones anglosajonas desde hace varios años en su estructura técnica, compuesta de titular y cuerpo. Por otra parte, estaría la ficha técnica, que teóricamente forma parte de la estructura de la crítica. De hecho hay varios autores que hablan de crítica deportiva refiriéndose a la crónica. Sin embargo para otros como Martínez Albertos la crítica debe reservarse a aquellas actividades humanas que encierran cierta dimensión cultural e ideológica y el deporte no se encuentra entre ellas. En el mundo deportivo señala Martínez Albertos hay comentaristas, reporteros y cronistas. ${ }^{6}$

\section{Lenguaje en el periodismo deportivo}

El deporte se ha convertido en una actividad dominante y ha difundido su idioma por todo el mundo. Ese lenguaje no obstante viene dado de las voces que configuran sus reglas y éstas son tributo en gran parte de su origen geográfico donde salieron: Las Islas Británicas. Por ello el lenguaje deportivo en su mayoría se escribe y pronuncia en inglés. En el caso de España se ha intentado españolizar esas palabras sin resultado salvo en algunos aspectos. El problema estriba en la difícil traducción de alguna de ellas, de forma que en gran número de disciplinas deportivas, toda o casi toda su terminología es inglesa, como es el caso del fútbol. Por otro lado la reconversión de muchas palabras no tuvo éxito. El sonido de la pronunciación ha sido el que ha sustituido a la palabra. Este es el caso del fútbol (balompié) y voleibol (balonvolea).

La utilización de las frases hechas procedentes de vocablos de diferentes idiomas y significados sólo se comprenden dentro de una conversación o exposición deportiva, pues al formularla en un contexto diferente no tendrían sentido. El

6 MARTÍNEZ ALBERTOS, J.L., Curso general de redacción periodística, Ed.revisada, Madrid, 1992, p.346. 
periodista deportivo debe ser cauto con su utilización y no usarlas constantemente. Para Alcoba las frases hechas son un recurso periodístico, una especie de "reserva periodística" para ser aprovechadas en momentos de agobio como cuando la crónica o comentario deben improvisarse y no se haya podido efectuar un análisis o las líneas a enviar son escasas (el espacio). Pese a que las crónicas y comentarios hayan perdido gran parte de su calidad literaria, de vez en cuando surgen términos explicativos de determinadas acciones, con calificaciones extraídas de otros contextos que mezclándose con los vocablos deportivos, concretan una frase inteligible si se conoce la temática deportiva a la cual se refiere. ${ }^{?}$

Al ser, la actividad deportiva "un enfrentamiento entre personas" se utiliza a menudo una terminología sacada del lenguaje militar. En una sociedad capitalista una de las posibilidades de dar salida a los distintos guerreros del hombre es el deporte. El deporte defiende una filosofía educativa, y lejana a cualquier espíritu provocador de conflictos. Su aspiración es la unidad y la paz entre los hombres, pero la competición deportiva incita al enfrentamiento.

El deporte en general y el fútbol en particular han generado un lenguaje propio con variantes lingüísticas y simbólicas, que a su vez han enriquecido el lenguaje del hombre. Es decir, se originaron y han ido generando una serie de expresiones y vocablos que con el tiempo se han multiplicado y expandido, formando un idioma particular de la actividad deportiva. Incluso el lenguaje creado por el deporte para definir sus múltiples modalidades y especialidades. Es tan rico que se han llegado a crear hasta diccionarios para conocer sus significados. Es el caso del diccionario creado por Acisclo Karag. Las acciones del deporte, producto del comportamiento de los jugadores y de los reglamentos que lo ordenan dan lugar en ocasiones a la extrapolación de sus significados a otras actividades. El deporte ha configurado un idioma cuyo lenguaje se proyecta desde diversos ángulos, ya sea hablado, escrito, simbólico o semiológico. ${ }^{8}$

La agencia EFE en sus publicaciones Manual de Español Urgente y El idioma español en el deporte extrae una serie de consideraciones básicas con respecto al lenguaje en sus secciones deportivas. Así no es partidaria de la innovación expresiva o los rasgos coincidentes con lo épico que se observa en la mayor parte de las crónicas deportivas. Como normas más destacadas en lo que concierne al lenguaje deportivo estarían según este manual evitar las frases hechas, las expresiones tópicas que, si bien un día fueron hallazgos de indudable valor comunicativo, hoy, ya desgastadas, entorpecen la comunicación y desinforman más que informan. Para lograr ésto, EFE establecerá una lista de ellas consideradas tabú para y por sus redactores:

7 ALCOBA, Antonio: Op. cit., p.155.

8 AlCoBA, A: Deporte y Comunicación. Dirección General de Deportes de la Comunidad de Madrid, Madrid. 1987. pp. 155 y 156 
"Acoso incesante sobre la meta contraria", "Cero a cero, empate sin goles", "Colocar el balón entre los tres palos", "Dar la vuelta al marcador", "El resultado campea en el marcador,... ${ }^{9}$

En relación con los extranjerismos, en la mayor parte ingleses en el caso del fútbol por su procedencia geográfica e histórica, EFE recomienda que se evite cuando haya expresión española que pueda remplazarlo. Un ejemplo puede ser la palabra "amateur", que podría sustituirse fácilmente por aficionado o no profesional. Lo mismo ocurre con "dribbling", pudiéndose decir regate o finta. Otro término muy utilizado es el de "mister", en vez de entrenador, preparador o técnico.

En cuanto a la redacción de los textos en la prensa escrita deportiva la agencia EFE se ajusta a una serie de criterios perfectamente definidos y específicos. Las noticias no deben sobrepasar las sesenta líneas o las seiscientas palabras, aproximadamente. Los titulares han de ceñirse a los sesenta y tres caracteres máximos por línea. Lenguaje conciso y frases breves. Supresión de adjetivos que no sean fundamentales para la comprensión del texto. De esta manera al seguir este formato, resultará un estilo más cuidado, aunque debe tenerse en cuenta también el papel que desempeña dicha agencia a sus abonados que utiliza un estilo puramente informativo, esto quizás se aleje un poco de las crónicas deportivas realizadas por los cronistas enviados de los diferentes diarios.

\section{Análisis de las crónicas deportivas:}

\section{José Antonio Sánchez Araujo}

Periodista sevillano, nacido en Alcalá de Guadaira (Sevilla). Ha trabajado en diferentes medios, siempre como periodista deportivo, aunque haya hecho breves incursiones en otros géneros como local o taurina. Se inicia profesionalmente en el diario Pueblo, más tarde en Sur/oeste, La Hoja del Lunes, Diario 16 y por último el diario $A B C$ en su edición de Sevilla, en la que continúa actualmente.

Respondería al clásico modelo de cronista deportivo. Además de su trabajo en el medio escrito, le acompaña el de periodista radiofónico. Tras un periodo en la desaparecida emisora La Voz del Guadalquivir, se consolidaría como periodista en la cadena SER en su emisora local de Sevilla, donde se encarga de seguir a los equipos de fútbol sevillanos Sevilla F.C. y Real Betis Balompié en sus desplazamientos fuera de la ciudad hispalense. Precisamente estos partidos que narra en radio, serán los que luego escribirá en forma de crónica para el diario $A B C$, una vez concluido el encuentro. Da entonces las últimas notas a su reportaje del partido, que además de la crónica incluiría comentarios y entrevistas ("Vestua-

9 Agencia Efe. El idioma español en el deporte. Efe, Logroño, 1992, p. 19. 
rios"), mandándolos posteriormente a la redacción a través de ordenador portátil y vía teléfono móvil. Ha colaborado además con diferentes publicaciones deportivas, casi siempre de fútbol como la revista El Sevillista o Mundo Bético y dirige además un programa radiofónico en la emisora local de Radio Sevilla en su banda de FM La cámara de los balones. Seguramente pocos periodistas sevillanos no sólo saben de fútbol como él sino que además conoce a fondo detalles casi desconocidos. Además destaca sobre todo su lenguaje sencillo, casi de la calle, aclarando todo aquello que pueda ofrecer la mínima duda tanto para el radioyente como para el lector de sus crónicas escritas.

Crónicas seleccionadas para el análisis de los siguientes partidos de fútbol, Diario $A B C$ de Sevilla (englobadas en el suplemento Campeón sí corresponden al lunes):

Valladolid - Sevilla (11/3/91), Bilbao - Betis (19/1/98), Oviedo - Betis (1/ 2/98), Elche - Sevilla (9/2/98), Zaragoza - Betis (16/2/98), Badajoz - Sevilla (23/ 2/98), Compostela - Betis (1/3/98).

Junto a estas siete crónicas se completará con una mención de otras correspondientes a etapas anteriores en concreto del diario Pueblo, Sur/oeste y La hoja del Lunes. Igualmente se utilizará un ejemplo anglosajón utilizado actualmente en las crónicas deportivas del periódico deportivo irlandés The Title la crónica Everton vs Liverpool (19/10/97).

Sería realmente difícil delimitar lo que ocuparía la crónica en sí del resto de la información deportiva, en este caso correspondiente al partido de fútbol y todo lo que le rodea. Teniendo en cuenta todo lo dicho anteriormente, la crónica sería sólo aquello correspondiente a narración del hecho de forma continuada; es decir, el partido con los momentos más importantes de la competición. Es un género de carácter informativo y personal, o sea narrado por un testigo o cronista.

Un cronista como José Antonio Sánchez Araujo. Actualmente escribe en el diario $A B C$. Su labor se centra en revisar aquellas partes y circunstancias del partido que justifican el resultado final, a juicio del cronista, basándose en una serie de datos. De esa manera cumple la denominada continuidad característica en este género, en lo que se refiere a la regularidad ofrecida en el tema y medio informativo. En este caso, cada vez que juegan Sevilla y Betis fuera de sus estadios, el lector del diario "ABC" de Sevilla encontrará dichas crónicas al día siguiente. Esas crónicas realizadas por un entendido en el tema como es Sánchez Araujo, buen conocedor no sólo de fútbol, sino también de los lugares desde donde se desarrollan los partidos, contextualizan los escenarios en los que se sitúan los partidos. En suma, estaríamos ante el prototipo de cronista, cuya firma supone un precinto de garantía para el lector.

Es importante destacar junto a la crónica en sí una serie de elementos, que 1 complementarían de una manera más objetiva la narración del relato en sí. Por un lado la denominada Ficha técnica, que según el Libro de estilo de ABC 
constituiría " un bloque de datos prácticos que encabeza a menudo críticas taurinas, de cine, teatro, fútbol,...", en el que " se resumen los datos básicos del espectáculo en cuestión, sus resultados escuetos o una breve reseña argumental" 10 .

En este sentido, los datos aportados serían los siguientes:

En primer lugar encabezaría los dos equipos que se enfrentan y el resultado final, debajo normalmente sobre un gráfico de un terreno de juego de fútbol, irían las aliniaciones de los equipos junto a las sustituciones producidas y en el momento realizado (Ej: Garitano, m.52). Por último, en la parte más bajairía el dato del colegiado o árbitro del partido, además de las tarjetas sacadas, a qué jugadores y en que momento. Se terminaría con los goles, si los hubiese, su autor y minuto. En ocasiones en la ficha técnica no aparece el gráfico ilustrativo y se limita tan sólo a las aliniaciones.

En cuanto a la estructura, siguiendo al Libro de estilo de $A B C$, se compondría en recuadro y en cuerpo menor que el general del periódico, apareciendo en negrita las primeras palabras, en este caso el resultado del partido.

Junto a la ficha técnica encontraríamos también al lado de la crónica, el bloque que $A B C$ denomina Así jugaron, el cronista valora en una frase la actuación de cada uno de los jugadores del equipo local, en este caso Sevilla o Betis, además de puntuar del 0 al 3 a cada uno de ellos. En ocasiones aparece bajo este bloque la denominada La Figura o El mejor, destacándose aparte la actuación decisiva de uno de los jugadores en el desarrollo del partido.

En el total de la información correspondiente a cada partido de fútbol, entre la que se englobaría como parte de ella la propia crónica y los bloques anteriormente señalados, habría que añadir la sección denominada Vestuarios, en el cual se complementaría a la crónica con un análisis de los propios protagonistas del evento, desde jugadores hasta entrenadores u otros personajes si los destaca José Antonio Sánchez Araujo. Tal como me comentó el propio cronista, esta parte se elabora desde el momento mismo que termina el partido y en su posterior rueda de prensa y entrevistas, poniéndose punto y final al hecho acontecido.

Por último destacar, por supuesto, las fotografías, que complementarían igualmente al conjunto de toda la información del partido, en especial si el contenido lo requiere, como sería el caso del partido Zaragoza - Betis, en el que a juicio del cronista el principal protagonista fue el árbitro Iturralde, colocándose incluso en la propia portada de la sección de deportes del diario $A B C$ su figura. Toda la información del partido, desde la crónica y los demás elementos que conforma el reportaje estaría incluido en la denominada sección de deportes del diario $A B C$, que si coincide con el lunes iría en el suplemento denominado Campeón.

10 Libro de estilo de ABC. "Normas de redacción y estilo de ABC". Prensa Española, Madrid, 1990. 
Centrándonos en la crónica en sí, las seis analizadas de $A B C$ comparten prácticamente los mismos elementos. En primer lugar la parte del título con el titular y subtítulo:

-El Titular precedería la crónica, en el que en una frase el cronista condensaría lo que ha supuesto el partido teniendo en cuenta todo lo que sucede en el mismo:

"El Sevilla regaló el partido en el segundo tiempo" ; "El Betis resistió, con uno menos el empuje del Athletic en San Mames" ; "El Betis dejó en Oviedo dos puntos" ; "Cinco meses después, el Sevilla vuelve a ganar fuera y a confiar en sí mismo" ; "El Zaragoza se alió con Iturralde para seguir siendo la bestia negra del Betis" ; "El Sevilla volvió a dar la decena fuera y ante miles de seguidores"; "Alfonso resolvió cuando peor lo pasaba el Betis"

El título suele ser lo primero que se lee sobre la crónica, de ahí la importancia que tiene para el cronista elegir la envoltura de lo que a continuación desarrollará con más detalle. Igualmente puede apreciarse perfectamente la enorme carga subjetiva del titular, aunque a diferencia de la prensa deportiva especializada suele ser menos sensacionalista el que nos ocupa.

-En cuanto al Subtítulo, constituye un punto y seguido del titular. En cuatro o cinco líneas se señala lo más sobresaliente del partido, considerándose sus claves principales. Según el Libro de estilo de $A B C$ este primer párrafo, entradilla o lead, contendrá los máximos datos de interés y justificación, que serán desarrollados en el resto de la información. ${ }^{11}$

Es decir si en un partido se ha destacado el nombre de un árbitro como principal protagonista, en el subtítulo se justifica él por qué de dicha circunstancia:

“...la actuación del colegiado Iturralde González, quien en las jugadas claves del partido perjudicó al Betis, al anular dos goles a Oli e Iván Pérez, dar penalti en una caída de Pier ante Finidi y expulsar a dos jugadores béticos".

- En cuanto al desarrollo exhaustivo de lo acontecido en cada uno de los partidos, que sería realmente el cuerpo de la crónica, se procedería al relato narrativo de lo principal del evento. En este caso suele ocupar gran parte de una página, a tres o cuatro columnas. Respondiendo a lo señalado en la primera parte, aquí se daría la narración de un hecho de forma continuada, es decir, un partido de fútbol.

No hay una estructura interna fija en general, sino que en cada una de ellas, según como se haya desarrollado cada partido, tendrá un esquema diferente. A esta circunstancia habría que añadir el carácter personal de la crónica, que implicará libertad de estilo y técnica.

11 Id., p 19. 
Se narrarían los momentos más destacados y decisivos del partido, acciones más importantes, siguiendo un orden cronológico y apuntándose los minutos claves tanto en la primera como en la segunda parte.

Al ser crónicas para un periódico local, se hace hincapié en el equipo de donde es el diario, es decir, que se incida en la mayor parte del relato de los hechos en los equipos de Sevilla y el Betis.

Normalmente cada una de las crónicas suele ser una continuidad de partidos anteriores, de ahí que suela iniciarse ésta haciendo referencia a los mismos: "Había eliminado el Zaragoza al Betis hacía sólo sesenta y dos horas y la lógica revancha estaba servida" ; "Igual que una semana antes contra el Valladolid, fue el habitual suplente Cañas quien abrió el marcador..."; "Volvía el Betis a tierras vascas tras su contundente victoria de la Copa en San Sebastián”.

La sencillez y claridad en el lenguaje constituye uno de los elementos del estilo de estas crónicas, generalizable a la mayor parte del periodismo deportivo como se indicó anteriormente. En el caso de Araujo, no hay ninguna duda acerca de dicha sencillez linguística, empleándose unos términos fáciles de entender, sin demasiadas figuras retóricas. En cuanto al vocabulario deportivo, estaríamos ante el propio universo del fútbol, con sus voces características. Al ser una narración de un hecho que produce constantes acciones en el lance del juego, la utilización de verbos es lo dominante, que además proporciona viveza al relato:

"Pronto empata el Betis, que juega mejor y domina al Zaragoza en un magnífico pase de Jaime que cabecea Alfonso a la red" ; "Nando cometió un error y dio paso a un saque de esquina que todos se tragan, portero incluido, y Carlos Torres de cabeza hace el gol”; "El Sevilla se adueñará del centro del campo y tendrá todo el terreno para que Tsartas empiece a colocar pases profundos en busca de la sentencia del partido”.

Como actividad deportiva, cada partido de fútbol supone un enfrentamiento entre dos rivales. En este sentido la terminología belicista que no escapa tampoco de estas crónicas, siendo muy abundante su presencia:

“...la lógica revancha estaba servida,” ; “...agarra un zurdazo impresionante que entra como un obús completamente imposible para el guardameta Prats".

- Deteniéndonos en cada una de las crónicas seleccionadas, la correspondiente al Valladolid-Sevilla del año 1991, en general se siguen las líneas señaladas anteriormente:

El relato poco varía del utilizado actualmente y tan sólo se observan alteraciones gráficas en la forma de la ficha técnica limitada sólo al texto.

Al considerar la clave del partido en una jugada del segundo tiempo, iniciará a partir de ahí el desarrollo propio del relato: 
"Ello provoca la desesperación de un equipo, al que le va a costar esa jugada el partido por una serie de circunstancias fáciles de comprender en adelante".

Es más que evidente su conocimiento del fútbol y su técnica al refererirse a la "zona Maturana" o fuera de juego. El elemento valorativo y crítico se mezclará en alguna ocasión con datos ajenos al partido como: todo".

"Polster, en día de cumpleaños quiere resolver todo por su cuenta y lo falla

De igual forma valora negativamente si lo estima oportuno o pone en duda la posición de los jugadores en los sistemas:

"...y y nada es posible porque el equipo no existe, no está en el partido y ve como el tiempo es sólo juez que determina su derrota" ; "Ramón en esa zona no tiene su sitio por mucho que lo intente hasta la extenuación...”.

$\mathrm{Al}$ final como en casi todas las crónicas terminará valorando bajo su punto de vista:

"En Valladolid se ofreció una muestra de lo que no tiene, ni debe hacer un equipo que quiere llegar lejos".

-La crónica correspondiente al partido Ath.Bilbao-Betis, de todas las seleccionadas, es la de mayor extensión. Se pone de nuevo de manifiesto su conocimiento de los lugares a los que va a denominar "La Catedral" al referirse al estadio de San Mamés o "Los leones" al equipo bilbaíno. Además utiliza la primera persona, aunque lo hace entre líneas o a modo de paréntesis:

“-y después de ver las imágenes por televisión creemos que fue injusta la segunda tarjeta -".

La valoración que hace del partido, aunque mantiene un equilibrio, llegará a rozar en ocasiones lo inverosímil:

"El partido de San Mamés ha sido un encuentro de más contenido futbolístico que de calidad".

.En el partido Zaragoza-Betis, José A. Sánchez Araujo confeccionará una crónica que sigue el orden propio del partido, aunque el relato girará siempre en torno a la actuación del árbitro desde practicamente el inicio, dándose además la circunstancia que encabezará incluso la portada del suplemento "Campeón":

"Iturralde la armó".

El Titular de la crónica continuará en esta misma línea:

"El Zaragoza se alió con Iturralde", avisándonos de la trascendencia en el partido. Nada más iniciarse el relato comenzará ya a referirse al mismo:

“... a los siete minutos y comienza la polémica actuación de Iturralde González cuando se anula...”, y no abandonará esta línea en toda la crónica:

“En esta jugada Iturralde no ha visto tampoco el codazo de Sundgen...”. 
A medida que se van narrando las acciones y lances más importantes del partido y jugadas más polémicas, se llegará en ocasiones a rozar un alto grado de subjetividad:

"Hay tardes que nada te sale y todo te perjudica, y para colmo el contrario está bendecido...".

La presencia de frases hechas, de origen belicista, habituales del lenguaje deportivo, no faltarán en esta crónica. Igualmente la claridad y sencillez en el léxico será la nota dominante, llegándose a emplear términos incluso coloquiales ("El pique de Radimov"), siguiéndose de esta manera lo que señalaba Alcoba sobre la simpleza que busca el lector de crónicas deportivas, que por lo general suele ser masivo. Mucho del relato, al igual que gran parte de todas las crónicas actualmente, se apoyará en la televisión, que es un elemento definitivo a la hora de juzgar determinadas jugadas, convirtiéndose casi en un evaluador de los arbitrajes.

Al final, a modo de epílogo, se destacará la máxima consecuencia del resultado:

"El Zaragoza, en menos de tres días, ha dejado al Betis fuera de la Copa del rey y le ha ganado los tres puntos ligueros".

Apreciamos igualmente expresiones técnicas de fútbol, que sólo tienen aquí sentido:

"El Zaragoza jugará a gusto, como sucedió en la Copa del rey, tocando mucho, encerrándose detrás y esperando los espacios que el Betis estará obligado a dejar por tener que jugar al ataque y practica las contras".

- De la crónica del Oviedo-Betis de nuevo vemos los apuntes técnicos como nota dominante. El relato girará en torno a la posible victoria del Betis que al final se redujo en un empate a cero, justificándose dicho resultado, sobre todo destacando la actuación del portero ovetense.

Respecto a los apuntes técnicos podría destacarse entre otros:

"Dominó la pelota, la tuvo más tiempo que el rival pero tanta exhibición de técnica, no le llevó a grandes sitios" ; "El cuadro verdiblanco careció de las bandas en la primera media hora de juego...” ; “...en la primera jugada del tiempo complementario Gamboa amenazó con dejarlo sin cabeza tras hacerle una corbata, que Esteban sacaría apuradamente junto al palo”.

El lenguaje seguirá una vez más cargado de expresiones metafóricas:

"El Oviedo encontró que le sonaba la flauta de tres contras...” ; “... a punto estuvo el Betis de quedarse sin el menguado botín de un punto” ; “...al cuarto de hora ya coleccionaba tres sustos".

La frase final una vez más, servirá para concluir con lo que a juicio de Araujo ha sido el partido:

"Era un partido para ganarlo y sólo empató. Menos es nada."

Otros detalles, como la ficha técnica aparece encabezando la crónica, de menor tamaño de lo que suele ser ahora habitual y sin gráficos. 
Por último, mencionar cómo la actualidad del momento romperá incluso el desarrollo normal del relato, convirtiéndose hasta en desgraciado protagonista en el propio evento deportivo:

"En el Carlos Tartiere se rezó una oración por las almas de Alberto Jiménez Becerril y Ascensión García” ; "En los prolegómenos del encuentro se rezó una oración por las almas de Alberto Jiménez Becerril y Ascensión García -gritos de Eta asesina atronaron el Tartiere-, vilmente asesinados en Sevilla, y los jugadores del Real Betis lucieron brazaletes negros en señal de luto."

- El partido Elche-Sevilla, también en el diario $A B C$, tendrá una crónica que en general continúa en la línea de las anteriores. Nuevamente, el titular servirá como punto de partida de lo que será la tónica del relato, en este caso el hecho de que el Sevilla gane tras un largo periodo:

"Cinco meses después, el Sevilla vuelve a ganar fuera y a confiar en sí mismo"

Además de los apuntes técnicos como nota dominante, se destacará a un jugador como clave en el desarrollo del partido, Tsartas. Por lo demás, al igual que en la mayor parte de sus crónicas, comenzará con el antecedente, de lo que podría considerarse la "precrónica" o lo anterior a lo que más tarde ocurrirá:

"Habían ganado el sábado los primeros clasificados, Las Palmas y Rayo Vallecano y empatado el Deportivo Alavés..."

Se comentarán igualmente las acciones más destacadas a juicio de Araujo acontecidas en el partido con los consiguientes análisis críticos.

En cuanto al léxico, reseñar de nuevo la presencia de frases hechas, muy habituales en el periodismo deportivo:

"No hay mejor forma de llegar al descanso, con un gol favorable en el tanteador" ; "Hay victorias en fútbol que tienen un gran valor..."

- En cuanto a la crónica del partido Badajoz-Sevilla el resultado del partido se analiza, planteándose una especie de investigación:

"Sería digno de un estudio sociológico-deportivo los comportamientos del conjunto sevillista."

Por otro lado la descripción habitual realizada del ambiente del partido, en consonancia con la derrota del marcador final, que se irá creciendo a medida que avanza, llegándose al clímax en el segundo gol del Badajoz:

"El ambiente, enrarecido de principio por la polémica del día del partido,...” ; “...mientras que los aficionados sevillistas, tristes y cabizbajos, abandonan las instalaciones de El Vivero".

En la parte final, a modo de conclusión, se valorará la derrota con apuntes técnicos:

"Errores en meta, solidez sólo en el centro de la zaga, más voluntad que aciertos en la salida de los laterales,..." 
- Para cerrar con el bloque de las crónicas del diario ABC, en el partido Compostela-Betis, al ser un encuentro en el que se dan cinco goles, el relato se caracteriza por ser más vivo, con constantes acciones y apuntes técnicos, y prácticamente centrada de principio a fin en el juego.

Muestra de esta viveza podía ser:

"El roteño, que había roto en velocidad sin balón y se había metido entre la zaga compostelana, reforzada con dos pivotes defensivos, remató el primer toque, sin dejarla caer, un centro medido de Fernando desde la izquierda y batió al meta Rafa con un perfecto giro de tobillo".

La narración del partido expuesta mantiene incluso la tensión hasta el final en el lector:

"Y cuando peor se le ponía el partido a un Betis completamente desbordado, surgió la calidad individual..."

- Una vez analizadas las crónicas del diario $A B C$, es interesante comparar las crónicas de José A. Sánchez Araujo de etapas anteriores en otros diarios, apreciándose su evolución como cronista, siempre de fútbol y de equipos sevillanos. En este sentido, volviendo veintiún años atrás, en el diario Pueblo, encontramos una breve crónica, en parte debido a la poca trascendencia del partido, Sevilla At. contra el Algeciras, correspondiente a la tercera división. Sin embargo, se observa ya prácticamente el mismo esquema utilizado posteriormente:

Tras el titular (El Sevilla Atlético contra el reloj), encontraríamos claramente tres partes diferenciadas, la primera situaría el contexto del partido (Encuentro correspondiente a la última jornada de la primera vuelta...), a continuación las alineaciones de los equipos y goles, que podría considerarse la ficha técnica, y por último en veinte líneas un resumen de lo más destacado del encuentro.

- Otra de las publicaciones en las que ha escrito Araujo crónicas sería la desaparecida Hoja del Lunes, en la que al igual que en $A B C$, será el enviado especial de los equipos sevillanos de fútbol. Hemos escogido dos ejemplos. En el primero de ellos, correspondiente a un partido entre el Barcelona y el Sevilla, se puede observar un esquema de crónica tal vez atípico con respecto a la línea general suya. Probablemente se incluye un exceso de subjetividad al criticar tanto al equipo rival e incluso a su ciudad, llegándose a implicar demasiado, pasando a participar en el relato como un personaje más, sobre todo en el inicio:

"Cuando esta mañana leía los periódicos de Barcelona pensé que estaba todavía dormido. Me froté los ojos y vi la triste realidad..."

Aunque a medida que avanza el relato se irá centrando más en lo que sería el juego en sí. Por otra parte, las alineaciones y lo que normalmente sería la ficha del partido, aparecen al final de la crónica, contra lo que se suele hacer habitualmente :

"Para los amantes de las estadísticas estas fueron las alineaciones a las órdenes del murciano Jiménez Madrid,...". 
De la misma publicación del mismo año, dos meses más tardes, comentar la crónica realizada del partido Las Palmas-Betis. En la misma sí encontramos la estructura habitual seguida en el resto de las crónicas suyas, con dos claros bloques diferenciados:

En el primero de ellos, contextualiza el espacio ("Magnifica entrada de público en el estadio Insular, casi lleno absoluto en noche fresquita y terreno de juego en irregulares condiciones"), alineaciones de los equipos, árbitro y valoración de su actuación, cerrando con un apartado de Incidencias.

El segundo bloque ocuparía lo que sería realmente el cuerpo en sí de la crónica, en la que se observa al igual que en el caso anterior un cierto exceso de valoración.

En comparación con las crónicas del diario $A B C$, se observa además de un mayor grado de subjetividad, un lenguaje incluso más popular("Lamo Castillo se hizo el sueco"), y en general un relato menos detallado en cuanto a contenido deportivo en sí. Habría también que hacer referencia a las crónicas del diario Sur/ oeste en las que Sánchez Araujo también realizó crónicas, aunque se sigue prácticamente un esquema similar a lo que más tarde realiza en el Diario 16 y $A B C$, aunque con menor extensión. Como novedad aportaría por primera vez las puntuaciones de los jugadores, valorándolos del uno al tres, en un bloque denominado Uno por Uno, y el apartado Responda Usted, en el que se entrevista al entrenador o protagonista del partido.

- Por último, comparando con las crónicas extranjeras y en concreto la anglosajona, se observa que el esquema seguido, es el mismo. De hecho como se dijo anteriormente el patrón de crónica empleado en nuestro país procede de dicho modelo. En este sentido, sirva de ejemplo una crónica del fútbol inglés, Everton-Liverpool, correspondiente al suplemento deportivo The Title del periódico Ireland on Sunday:

El titular, al igual que en las crónicas anteriores, muestra lo que va a ser la crónica del relato:

Happy days are here again, refiriéndose al buen momento tras un largo periodo del Everton.

Tras el titular aparece el nombre o firma del cronista junto a su fotografía, Aidan FitzMaurice, encargado en este periódico del partido del día en la Liga Inglesa de Fútbol, en este caso el clásico derbi de los equipos de Liverpool.

El resultado final del partido Everton 2 - Liverpool 0, encabezaría la crónica en sí. Al igual que el caso de Araujo, se observa claramente un estilo personal, en el cual se valora y crítica constantemente y en el que son constantes los apuntes técnicos de fútbol. Destacar del mismo modo, la alusión a la televisión para destacar alguna jugada.

En cuanto al lenguaje, pese a que estemos en otro idioma, se siguen utilizando frases hechas, metáforas y juegos de palabra, propios del lenguaje deportivo: 
“...he didn't want the phrase A Neil Ruddock to enter the Chambers Dictionarity as a synonym for own goal".

Completando el relato del partido, estarían por un lado La Ficha Técnica", denominada The Team Sheet donde se puntuaría también la actuación de los jugadores de los dos equipos. Y la figura del partido, Man of the Match, en la que se destaca su actuación decisiva en el resultado final del partido.

A modo de reflexión sobre la crónica deportiva, exponer las siguientes consideraciones:

El originario carácter polisémico de la crónica persistirá como género periodístico, lo que ha generado confusión a la hora de definirlo. En el ámbito general consideraríamos la crónica como una narración de unos hechos noticiosos, ocurridos en un periodo de tiempo, por un cronista que los ha vivido de cerca como testigo directo que a la vez analiza e interpreta mediante una explicación personal. Tiene un carácter ante todo, informativo, aunque sin olvidar el elemento personal. La firma del cronista llega en ocasiones a convertirse en el certificado de calidad de la propia información. El caso de José Antonio Sánchez Araujo además de ser testigo directo de lo que ocurre en cada partido, se da incluso la circunstancia que narra en radio el mismo evento, lo cual le confiere más confianza al lector, que ha podido ser antes también oyente suyo.

La crónica deportiva constituye un buen ejemplo de la necesidad de conocimiento sobre aquello que se cuenta, no es suficiente que le agrade al periodista sino que hay que saber realmente sobre él. En prensa escrita, este género ha evolucionado tanto hasta el punto de convertirse en los últimos años en complemento de los otros medios. La presencia de la televisión y su enorme trascendencia en el fútbol ha hecho cambiar la propia forma de la crónica en la prensa escrita, haciéndose constantes alusiones al medio audiovisual.

Sencillez y claridad en el lenguaje pero a la vez precisión en lo que se cuenta será una de las constantes en el estilo de las crónicas futbolísticas, que suele ser un tipo de información dirigida a toda clase de público masivo. Por último no hay que olvidar una importante función del cronista deportivo, que no es otra que la de educar al público en los aspectos técnicos del deporte y sus reglas, y a la vez rechazar y criticar aquello que se salga de lo puramente deportivo, como son los actos vandálicos, no respetar al rival ni las reglas del juego,... 